\title{
PROTON DECAY IN SUPERSYMMETRIC MODELS
}

\author{
Savas DIMOPOULOS ${ }^{1}$ \\ Department of Physics, University of Michigan, Ann Arbor, MI 48109, USA \\ and Department of Physıcs, Stanford University, Stanford, CA 94305, USA
}

\author{
Stuart RABY \\ Los Alamos Scientific Laboratory, Los Alamos, NM 87545, USA \\ and \\ Frank WILCZEK \\ Institute for Theoretical Physics, Santa Barbara, CA 93106, USA
}

Recelved 2 December 1981

\begin{abstract}
We discuss proton decay in supersymmetric theores We find that it is possible to obtain rates which are comparable with those of standard SU(5). In the presence of a discrete symmetry which occurs in an SU(5) supersymmetric unified model we obtain a definite prediction for the dominant decay mode, $1 \mathrm{e} p \rightarrow \mathrm{K}^{+} \bar{\nu}_{\mu}$ and $\mathrm{n} \rightarrow \mathrm{K}^{0} \bar{\nu}_{\mu}^{-}$.
\end{abstract}

1. Introduction. Supersymmetry has recently been proposed as a possible solution to the gauge hierarchy problem [1]. It has been shown that in supersymmetric unified models (SUM) the unification scale moves up to $10^{16-17} \mathrm{GeV}$ [2]. Thus the standard processes leading to proton decay via tree diagrams result in unobservably small rates. Recently, Weinberg has shown that one-loop effects can dominate over tree results leading to larger baryon-number violating amplitudes [3].

In this paper we discuss proton decay in SUMs. In section 2 we discuss the general framework on which our results are based. In section 3 we discuss proton decay in models containing a discrete symmetry which occurs in an SU(5) SUM [4]. In this class of models we obtan a definite prediction for the dominant decay modes of the proton and neutron, i.e. $\mathrm{p} \rightarrow \mathrm{K}^{+} \bar{\nu}_{\mu}$ and $\mathrm{n} \rightarrow \mathrm{K}^{0} \bar{\nu}_{\mu}$. The rates for these processes depend on the unknown masses of the gluino and scalar quarks. Reasonable values for these masses can yield lifetimes

${ }^{1}$ Present address' Harvard University, Cambridge, MA 02138 , USA. on the order $\sim 10^{30} \mathrm{yr}$. In section 4 we discuss proton decay in a more general context. We find that both the rates and final states for proton decay are then very model dependent. These cases, however, exhibit the interesting decay modes $\mathrm{p} \rightarrow$ photino $+X$ and $\mathrm{p}$ $\rightarrow$ goldstino $+\mathrm{X}$.

2. General considerations. We consider SUMs in which the gauge symmetry between $10^{17} \mathrm{GeV}$ and $300 \mathrm{GeV}$ is the standard $\mathrm{SU}(3) \otimes \mathrm{SU}(2) \otimes \mathrm{U}(1)$. We shall assume that the low-energy particles in the theory are the ordinary quarks and leptons, two Higgs doublet scalars, the gauge bosons; and their supersymmetric partners. In addition we allow for the possibility of having $S U(3) \otimes S U(2) \otimes U(1)$ singlet supermultiplets.

We shall use the following superfield notation. $\Phi$ is a left-handed chiral superfield which can be written in the component form

$\Phi=2^{-1 / 2} \phi+0 \psi+2^{-1 / 2} F \theta \theta$,

where $\phi, \psi$ and $F$ are the scalar, fermion and auxiliary fields, respectively. We then have the states 
$\Phi_{\mathrm{q}}^{l}, \quad \Phi_{\overline{\mathrm{u}}}^{l}, \quad \Phi_{\mathrm{d}}^{i}, \quad \Phi_{\ell}^{i}, \quad \Phi_{\mathrm{c}^{+}}^{i}$

where $q$ and $\ell$ denote the quark and lepton doublets and $i=1,2,3$ is a family index. We also have Higgs superfields $\Phi_{h}, \Phi_{\bar{h}}^{-}$where $h$ and $\bar{h}$ are the Higgs' with hypercharge +1 and -1 , respectively. $\Phi_{0}$ will denote singlet superfields.

In such theories there is a baryon-number violating dimension-four operator which will lead to an extremely short proton lifetıme Thus this operator must be forbidden by additional global symmetries. The dangerous dimension-four operator is

$$
\int \mathrm{d}^{2} \theta \Phi_{\overline{\mathrm{u}}}^{l} \Phi_{\mathrm{d}}^{\frac{j}{\mathrm{~d}}} \Phi_{\overrightarrow{\mathrm{d}}}^{k}
$$

As Weinberg has pointed out, in theories where this operator is forbidden by global symmetries, there can still exist dimension-five baryon-number violating operators [3]. These operators are

$$
\begin{aligned}
& \int \mathrm{d}^{2} \theta \Phi_{\mathrm{q}}^{l} \Phi_{\mathrm{q}}^{j} \Phi_{\mathrm{q}}^{k} \Phi_{\ell}^{m}, \quad \int \mathrm{d}^{2} \theta \Phi_{\overline{\mathrm{u}}}^{i} \Phi_{\overline{\mathrm{u}}}^{j} \Phi_{\mathrm{d}}^{k} \Phi_{\mathrm{c}^{+}}^{m}, \\
& \int \mathrm{d}^{2} \theta \Phi_{\mathrm{q}}^{i} \Phi_{\mathrm{q}}^{j} \Phi_{\mathrm{q}}^{k} \Phi_{\overline{\mathrm{h}}}, \quad \int \mathrm{d}^{2} \theta \Phi_{\overline{\mathrm{u}}}^{l} \Phi_{\overline{\mathrm{d}}}^{j} \Phi_{\overline{\mathrm{d}}}^{k} \Phi_{0}, \\
& \int \mathrm{d}^{2} \theta \Phi_{\mathrm{q}}^{l} \Phi_{\mathrm{q}}^{j} \Phi_{\overline{\mathrm{q}}}^{+}
\end{aligned}
$$

3. Famuly Reflection Symmetry (FRS). The family reflection symmetry defined by

$\Phi_{\mathrm{q}}^{i} \rightarrow-\Phi_{\mathrm{q}}^{i}, \quad \Phi_{\overline{\mathrm{u}}}^{\underline{l}} \rightarrow-\Phi_{\overline{\mathrm{u}}}^{i}, \quad \Phi_{\mathrm{d}}^{\underline{l}} \rightarrow-\Phi_{\mathrm{d}}^{i}$,

$\Phi_{\ell}^{I} \rightarrow-\Phi_{\hat{\ell}}^{i}, \quad \Phi_{\mathrm{e}^{+}}^{i} \rightarrow-\Phi_{\mathrm{e}^{+}}^{i}$,

is a natural discrete symmetry in an SU(5) SUM [4]. It forbids the dimension-four operator of eq. (2.3). In addition it eliminates the dimension-five operators of eqs. $(2.4 \mathrm{c}-\mathrm{e})$. Thus the only dimension-five operators which contribute to proton decay are those of eqs. $(2.4 a, b)$.

As a result of Bose statistics for these scalar superficlds it is easy to see that both these operators vanish if there is only one family. Therefore the only relevant operators for proton or neutron decay are the following

$$
\int \mathrm{d}^{2} \theta \Phi_{\mathrm{u}} \Phi_{\mathrm{d}} \Phi_{s} \Phi_{\nu_{\mu}}
$$

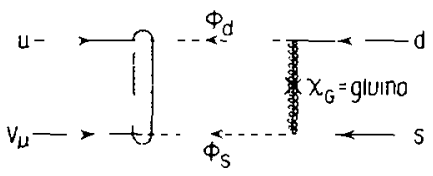

Fig 1 The dominant graph contributing to proton decay in theories with family reflection symmetry. The vertex on the left corresponds to the effective lagrangian (3 3). The fermion exchanged on the right is a glumo The cross on the gluino line represents a churality flupping Majorana mass

$$
\int \mathrm{d}^{2} \theta \Phi_{\mathrm{u}} \Phi_{\mathrm{d}} \Phi_{\mathrm{c}} \Phi_{\mu^{-}}, \quad \int \mathrm{d}^{2} \theta \Phi_{\overline{\mathrm{u}}} \Phi_{\mathrm{d}^{-}} \Phi_{\overline{\mathrm{c}}} \Phi_{\mu^{+}}
$$

As a result we will conclude that strange-particle final states will be dominant.

Let us consider the processes mediated by the operator of (3.2a). The effective lagrangian arising from this operator is

$$
\begin{aligned}
& G_{\text {eff }}\left[\mathrm{ud} \phi_{\mathrm{s}} \phi_{\nu_{\mu}}+\right.\text { permutations } \\
& \left.\quad+\phi_{\mathrm{u}} \phi_{\mathrm{d}} \phi_{s} F_{\nu_{\mu}}+\text { permutations }\right] .
\end{aligned}
$$

The first term provides the dominant contribution to proton decay via the graph of fig. 1 . This graph leads to the processes $\mathrm{p} \rightarrow \mathrm{K}^{+} \bar{\nu}_{\mu}$ and $\mathrm{n} \rightarrow \mathrm{K}^{0} \vec{\nu}_{\mu}$.

Let us now estimate the magnitude of this process and describe why we expect this to be the dominant mode. In our estimate we rely heavily on SU(5). In such a model the fermionic terms in the effective lagrangian (3.3) come from an exchange of a color triplet fermionic Higgs. As a result we find

$G_{\text {eff }} \approx g_{\mathrm{u}} g_{\mathrm{s}} / M_{\mathrm{h}_{3}}$,

where $M_{\mathrm{h}}$ is the mass of the color triplet Higgs and $g_{\mathrm{u}}, g_{\mathrm{s}}$ are Yukawa couplings (see fig. 2). In the limit $M_{\mathrm{h}_{3}} \gg \mu \gg m_{\mathrm{g}}$ (where $\mu$ is the scalar quark mass and $m_{\mathrm{g}}$ the Majorana part of the gluino mass) we obtain the effective four-fermion operator

$G_{\text {SUM }}$ uds $\nu_{\mu}$

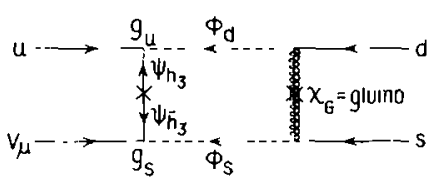

$\Gamma$ ig 2. The dominant graph contributing to proton decay in theones with famly reflection symmetry. The fermion exchanged on the left is a fermionic I liggs color triplet. The cross is the Dirac mass of this fermion Higgs 
where

$G_{\mathrm{SUM}}=\left(g_{\mathrm{u}} g_{\mathrm{s}} / M_{\mathrm{h}_{3}}\right)\left(m_{\mathrm{g}} / \mu^{2}\right) \alpha_{\mathrm{c}} / 2 \pi$.

This is to be compared with the standard SU(5) result

$G_{\mathrm{GUT}} \sim 10^{-30} \mathrm{GeV}^{-2}$

For reasonable values of the parameters in (3.5)

$M_{\mathrm{h}_{3}} \sim 10^{17} \mathrm{GeV}, \mu \sim 60 \mathrm{GeV}, m_{\mathrm{g}} \sim 1 \mathrm{GeV}$,

$\alpha_{\mathrm{c}} \sim 0.1, \quad g_{\mathrm{u}} \sim 4 \times 10^{-5}, \quad g_{\mathrm{s}} \sim 8 \times 10^{-4}$,

we find $G_{\mathrm{SUM}} \simeq G_{\mathrm{GLT}}$.

Several comments are in order. Why is this the dominant graph?

(a) Note that each vertex on the left side of fig. 2 contains transitions between members of the same family. Thus it is not Cabibbo suppressed.

(b) The intermediate state contains two colored scalar quarks. Therefore they can intcract with the exchange of a strongly interacting gluino.

(c) The graph of fig. 2 is proportional to Yukawa couplings because we have exchanged Higgs fermions. Naively one might expect that gauge fermions (e.g. the partner of the $\mathrm{X}$ or $\mathrm{Y}$ bosons) might give a larger result. However, an operator of the form

$$
\int d^{2} \theta \Phi^{4}
$$

can only be obtained by the exchange of matter fermions since each vertex flips chirality.

Now let us evaluate the contribution to proton decay coming from operators of the form

$G_{\mathrm{cff}} \phi_{\mathrm{u}} \phi_{\mathrm{d}} \phi_{\nu_{\mu}} F_{\mathrm{s}}$

[see eq. (3.3)]. In fig. 3 we give an exchange which results in the operator (3.9). Now using the low-energy Yukawa coupling

$g_{\mathrm{s}} F_{\mathrm{s}} \phi_{\mathrm{s}}^{-} \phi_{\bar{h}_{0}}$

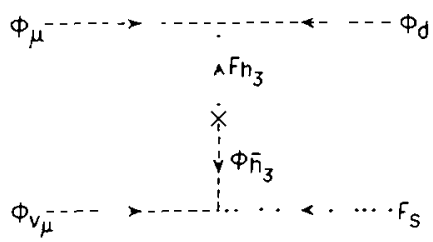

Fig. 3. Five scalar operator contributing to proton decay.

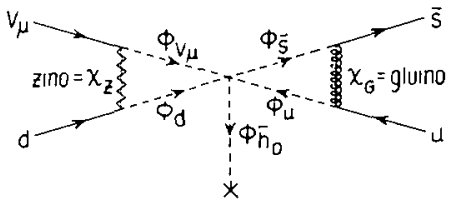

Fig. 4. How the five scalar operator of fig 3 contributes to proton decay. $X Z$ is the zion or the supcrpartner of the $Z$ boson.

we obtain the dimension-five, 5 scalar operator

$G_{\text {eff }} g_{\mathrm{s}} \phi_{\mathrm{u}} \phi_{\mathrm{d}} \phi_{\nu_{\mu}} \phi_{\overline{\mathrm{s}}}^{+} \phi_{\overline{\mathrm{h}}_{\mathrm{o}}}^{+}$,

This contributes to proton decay via the graph of fig.

4. As a result we obtain the same effective four-fermion operator as in (3.5) with a new Fermi constant

$G_{\mathrm{SUM}}^{\prime} \simeq\left(G_{\mathrm{cff}} V / \mu^{2}\right)\left(\alpha_{\mathrm{c}} / 2 \pi\right)\left(\alpha_{2} / 2 \pi\right) g$

where $V \equiv\left\langle\phi_{\bar{h}^{0}}\right\rangle$ and we have assumed that $m_{\mathrm{z} \text { no }} \lesssim \mu$. It is easy to check that for a value of $m_{\mathrm{g}}$ (the gluino mass) greater than $\sim 1 \mathrm{MeV}$ these graphs are not important compared to the graph of fig. 2 .

Finally the operators of eqs. $(3.2 b, c)$ are unimportant since they involve additional small couplings in order to take the charm quark into a strange quark.

We thus conclude that in theories with the FRS and with only standard low-encrgy particles, the dominant decay modes in baryon decay are

$\mathrm{p} \rightarrow \mathrm{K}^{+}+\bar{\nu}_{\mu}, \quad \mathrm{n} \rightarrow \mathrm{K}^{0}+\bar{\nu}_{\mu}$

We note, however, that in contrast to standard SU(5) the rate for these decay modes depends on unknown low-energy parameters of the theory e.g. $m_{\mathrm{g}}$ and $\mu$ [see (3.5)].

4. Other discrete symmetries. It is easy to find other symmetries which forbid the disastrous dimension-four operators of eq. (2.3) but allow the additional dimension-five operators of eqs. $(2.4 \mathrm{c}-\mathrm{e})$. Thesc operators can lead to different final states. Notice that they violate baryon number but not lepton number. As a result there will be the new decay modes

$\mathrm{p} \rightarrow$ photino $+\mathrm{X}, \quad \mathrm{p} \rightarrow$ goldstino $+\mathrm{X}$, which satisfy $\Delta B=1$ and $\Delta L=0$ (see fig. 5). We shall not even attempt to estimate the order of magnitude of these amplitudes since they depend essentially on unknown parameters of an unknown theory. 


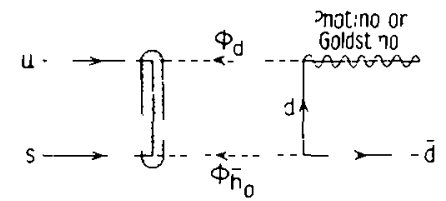

F1g 5. Proton decay medrated by the operator $(24 c)$

We also observe that in a theory which includes the dimension-four operator

$$
\int \mathrm{d}^{2} 0 \Phi_{\mathrm{d}}^{-} \Phi_{\mathrm{q}} \Phi_{\ell}
$$

which satisfies $\Delta B=0, \Delta L=1$ it is even possible to obtain a dimension-five operator which results in the standard dominant mode

$\mathrm{p} \rightarrow \mathrm{e}^{+}+\pi^{0}$

(see fig. 6). Finally we note that it is also possible to invent symmetries which forbid all the dimension-four and -five operators in eqs. (2.3), (2.4). Thus the dominant baryon violating operators in such a theory would be dimension-six operators which are unobserv-

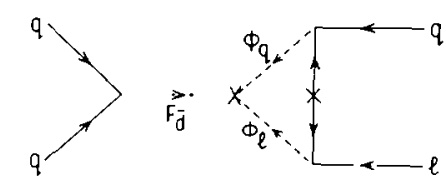

Fig. 6 Proton decay mediated by the operators $(2.4 \mathrm{e})$ and (4)). ably small. An example of such a symmetry is a new $\mathrm{U}(1)$ that appears naturally in $\mathrm{E}(6)$ and takes the same value on every member of a family.

5. Conclusion. The above analysis shows that one can not make definite predictions for the final states in proton decay in a model independent way. However, if the decay modes $\mathrm{p} \rightarrow \mathrm{K}^{+}+\bar{\nu}_{\mu}$ and $\mathrm{n} \rightarrow \mathrm{K}^{0}+\bar{\nu}_{\mu}$ are observed to be the dominant modes in baryon decay, then this will provide evidence in favor of supersymmetric theories with the discrete famly reflection symmetry.

\section{References}

[1] S Dimopoulos and S Raby, Supercolor, Stanford preprint (Tebruary 1981),

F Witten, Dy namical breakıng of supersymmetry, Princeton preprint;

M Dine. W. Fishler and M Srednickı, Supersymmetric technicolor, Princeton preprint.

[2] S Dimopoulos, S. Raby and F Wilczek, Scale of unification in supersymmetric models, Santd Barbdta preprint, N. Saka1. Tohoku University preprint TU/81/225

[3] S Weinberg, Supersymmetry at ordinary energies I, HUTP-81/^047, see also, N. Sakal and T Yanagida, Proton decay models Max Planck preprint (October 1981).

14] S. Dimopoulos and H. Georgi, Harvard preprint HUTP-81 $\Lambda 022(1981)$;

N P. Nulles and S. Raby, Supersymmetry and the strong CP problem. SLAC preprint;

Ł Witten, Dynamical breaking of supersymmetry.

Princeton preprint,

N. Sakdl, Tohoku University preprint 'I L $/ 81 / 225$ 